Check for updates

Cite this: RSC Adv., 2017, 7, 28733

\title{
Waste activated sludge (WAS) dewatering properties of an original hydrophobically modified polyacrylamide containing a cationic microblock structure $\uparrow$
}

\author{
Yuhao Zhou, (D) ab Huaili Zheng, ${ }^{\text {ab }}$ Baoyu Gao, (D) ${ }^{\mathrm{c}}$ Yingpeng Gu, ${ }^{\text {ab }}$ Xiang Li, ${ }^{\text {ab }}$ \\ Bingzhi Liu ${ }^{\mathrm{ab}}$ and Andrea Mavarro Jiménez ${ }^{\mathrm{ab}}$
}

Chemical conditioning, as one of the core technologies used for the dewatering pretreatment of sludge, can efficiently improve the dewaterability of WAS and hence reduce the expense of the transportation and disposal of WAS. Cationic polyacrylamide has been widely utilized as a chemical conditioner owing to its high performance and economic advantages. However, high-performance and economical flocculants are still needed. In this study, a novel hydrophobically associating polyacrylamide, which was denoted as TPADL and contained a cationic microblock structure synthesized by a UV-initiated template copolymerization technique, was employed in a dewatering test on WAS. The chemical composition of TPADL was confirmed via hydrogen nuclear magnetic resonance $\left({ }^{1} \mathrm{H}\right.$ NMR) spectroscopy and Fourier transform infrared (FTIR) spectroscopy. Moreover, ${ }^{1} \mathrm{H}$ NMR spectroscopy demonstrated a highly concentrated distribution of cations on the main chain. Furthermore, thermogravimetric analysis (TGA) was utilized to investigate the thermal stability of TPADL, and its apparent viscosity was also measured to evaluate its rheological characteristics. Dewatering tests found that the TPADL flocculant exerted a synergistic function owing to the cationic microblock structure and hydrophobic association and exhibited superior dewatering performance in comparison with normal random cationic polyacrylamide (CPAM). The filter cake moisture content (FCMC) and specific resistance to filtration (SRF) reached $64.98 \%$ and $1.3 \times 10^{12} \mathrm{~m} \mathrm{~kg}^{-1}$, respectively, for TPADL at the optimal dosage of $1.5 \mathrm{mg} \mathrm{g}^{-1}$ dry solids of WAS (calculated value). The floc size distribution revealed that hydrophobic association remarkably increased the floc size owing to enhancements in bridging absorption ability, whereas the cationic microblock structure helped form a denser and more compact floc structure, and the higher charge neutralization ability enabled an increase in floc strength. In addition, the synergistic function significantly improved the regeneration ability of flocs. Scanning electron microscopy (SEM) analysis indicated that the stronger floc structure could act as a skeleton to form pores and a channel-like structure, which made filter cake a favorable draining medium for the release of water and thus contributed to an increase in dewaterability. This study provided an analysis for the development of high-performance and economic flocculants by a combination of the controllable concentrated distribution of cations and hydrophobic association.

Received 11th March 2017 Accepted 10th May 2017

DOI: $10.1039 / \mathrm{c} 7 \mathrm{ra02939j}$

rsc.li/rsc-advances in pollutant removal made by current tightened regulations., ${ }^{1,2}$ The large quantities of WAS are a troublesome by-product of wastewater treatment plants (WWTPs), which need to be appropriately handled; otherwise, they could lead to serious environmental impacts. ${ }^{3}$ In China, the current production of dewatered WAS is greater than $3.0 \times 10^{7}$ tons (with a water content of $80 \%$ ) and is expected to continue to increase owing to the stricter environmental regulations and growing amounts of wastewater. ${ }^{4,5}$ The management of WAS represents a considerable portion of the total operating costs of WWTPs and also causes a great economic burden. ${ }^{6,7}$ To reduce the environmental and economic impacts of WAS, dewatering is a crucial step for reducing moisture content and sludge volume by solid-water
${ }^{a}$ Key Laboratory of the Three Gorges Reservoir Region's Eco-Environment, State Ministry of Education, Chongqing University, Chongqing 400045, China. E-mail: zhl@cqu.edu.cn; Fax: +86 23 65120827; Tel: +862365120827

${ }^{b}$ National Centre for International Research of Low-Carbon and Green Buildings, Chongqing University, Chongqing 400045, China

${ }^{c}$ Shandong Key Laboratory of Water Pollution Control and Resource Reuse, School of Environmental Science and Engineering, Shandong University, Jinan 250100, China

$\dagger$ Electronic supplementary information (ESI) available. See DOI: 10.1039/c7ra02939j 
separation, which is an effective approach for reducing the expense of the transportation and disposal of WAS. ${ }^{\mathbf{8 - 1 0}}$

With the aim of reducing moisture content, various treatments can be applied before the final disposal of WAS. ${ }^{11}$ Mechanical dewatering is mostly performed in medium/large WWTPs. ${ }^{1}$ Nevertheless, without pretreatment, the heterogeneous and stable colloidal nature of WAS may considerably hinder the dewatering process and fail to meet the requirements of subsequent treatment processes. ${ }^{\mathbf{1 , 9}}$

To enhance the dewaterability of sludge, various kinds of pretreatment process have been widely studied and developed, including thermal, chemical, magnetic, mechanical, acoustic, electric and hybrid pretreatment processes. ${ }^{12}$ Among these, chemical conditioning is one of the most efficient pretreatment processes for improving the dewatering performance of WAS. Cationic polyacrylamide, as one of the most widely applied chemical conditioners, can significantly improve the mechanical dewaterability of WAS by flocculating fine colloidal sludge particles to form giant flocs ${ }^{6,13}$ via the two extensively accepted mechanisms of bridging and surface charge neutralization. ${ }^{\mathbf{1 4}}$

However, traditional cationic polyacrylamides synthesized by common methods usually have a random distribution of cationic units along the polymer chain. According to Guan et al. and Chen et al., ${ }^{15,16}$ for such materials, owing to the dispersed arrangement of cationic units, charge neutralization cannot be fully utilized to neutralize the surface charge on particles. As shown in Fig. 1(a), many cationic units situated on loops and tails of the main chain are wasted. In contrast, the cationic microblock structure of the novel products synthesized by template copolymerization can provide stronger and less easily separated segments for absorption, as shown in Fig. 1(b), which lead to a stronger and more compact floc structure and hence better flocculation performance. Furthermore, the concentrated distribution of charge can provide a stretched linear configuration of the polymer chain in solution, which contributes to better bridging performance to form larger flocs, as was expected. ${ }^{17}$
Hydrophobic modification has been believed to be a promising way to enhance the performance of polymers. ${ }^{18,19}$ The incorporation of a small number of hydrophobic groups into a hydrophilic macromolecular chain imparts unique rheological characteristics. ${ }^{20}$ In aqueous solutions, hydrophobic groups on a polymer chain can aggregate together to form intra- and inter-molecular associations to minimize the exposure of the hydrophobic units to the solvent, as shown in Fig. 1(c). This kind of association, in particular, intermolecular association, can significantly increase the viscosity of the solution, as well as association reactions between hydrophobic groups along polymer chains and the hydrophobic constituents of WAS particles. ${ }^{21-23}$ Hence, the flocculation efficiency was improved.

The hydrophobically associating polyacrylamide with a cationic microblock structure denoted as TPADL was synthesized by UV-initiated template copolymerization of a cationic monomer (DAC), a hydrophobic monomer (LA) and acrylamide (AM). As can be imagined, both the cationic microblock structure and the hydrophobic groups were incorporated into the macromolecular polymer chain, and the stronger charge neutralization, bridging absorption, and hydrophobic association effects could together improve the flocculation performance during sludge dewatering treatment. Given that TPADL is supposed to provide better charge neutralization and bridging absorption performance, as well as interactions between the hydrophobic units of macromolecular flocculants and the hydrophobic components of fine colloidal particles in WAS, satisfactory efficiency in the solid-water separation of WAS would be achieved.

In order to decrease operating costs and guarantee the efficiency of dewatering to meet the needs of increasingly strict environmental legislation, it seemed essential and urgent to investigate the flocculation mechanism and floc properties in sludge dewatering. However, to the authors' knowledge, the employment of the novel high-performance TPADL in the dewatering of WAS has not yet been well studied. In this paper, the authors mainly focus on investigating the chemical properties of this novel TPADL, including determining its composition,
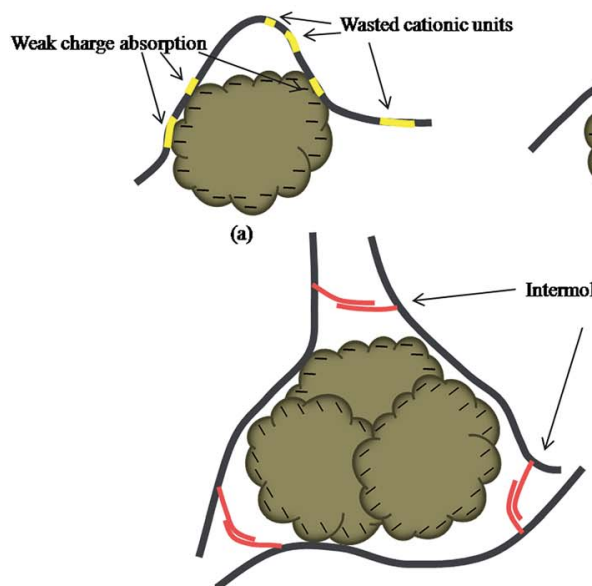

(c)

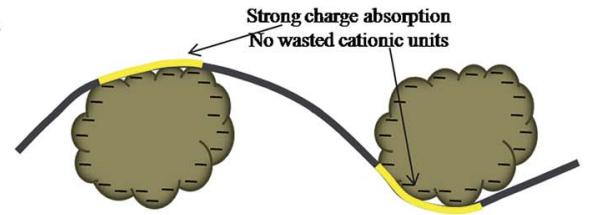

(b)

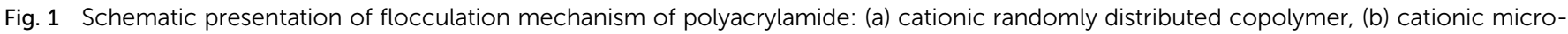
block copolymer and (c) hydrophobic association copolymer. 
structural characteristics and thermal stability by FTIR spectroscopy, ${ }^{1} \mathrm{H}$ NMR spectroscopy and TGA analysis. The rheological characteristics of this TPADL in solution were also investigated. The dewaterability of WAS using this novel flocculant was evaluated in terms of the FCMC and SRF. The zeta potential and floc properties in terms of size distribution, floc breakage and recovery factor and fractal dimension were further measured for the thorough study of the flocculation mechanism.

\section{Experimental section}

\subsection{Flocculants}

2.1.1 Preparation of TPADL. A template copolymerization technique was employed to synthesize hydrophobically associating TPADL with a cationic microblock structure via UV initiation. TPADL was prepared by copolymerization from monomers of AM (>99\%, industrial grade, Chongqing Lanjie Tap Water Company, China), DAC (80\% in aqueous solution, industrial grade, Guangchuangjing Imp. Exp. Co., Ltd., China) and LA (95\%, industrial grade, Hubei Jusheng Technology Co., Ltd., China), and PAAS (30\% in aqueous solution, industrial grade, Shandong Xintai Water Treatment, Zaozhuang, China) with a low molecular weight of 3400 was used as the template reagent. The other additional agents, including V-044 as a photoinitiator and Tween-80 as a non-ionic dispersant, were of analytical grade. Deionized water was utilized throughout this research. A predetermined amount of the monomers was placed in a highly transparent glass reaction vessel with the regular ratio of PAAS and other additional agents. After being purged for $30 \mathrm{~min}$ with an $\mathrm{N}_{2}$ stream for the removal of oxygen, the vessel was exposed to UV irradiation from a $1000 \mathrm{~W}$ highpressure mercury lamp (Shanghai Jiguang Special Lighting Electrical Plant, China) for a certain time at ambient temperature. After irradiation, the products were purified by acetone and ethanol and PAAS was also removed before the products were dried and granulated. ${ }^{15,16}$ More details of the synthesis process can be seen in our previous works. ${ }^{19,24-26}$ The proposed reaction route is outlined in Fig. S1 in the ESI. $\dagger$

2.1.2 Characterization of TPADL. The intrinsic viscosity was measured with an Ubbelohde viscometer (Shanghai Shenyi Glass Instrument Co., Ltd., China) by the one-point method with reference to the Solomon-Ciuta formula. ${ }^{18,27}$ The FTIR spectra of the products were recorded using $\mathrm{KBr}$ pellets with a 550 series II infrared spectrometer (Mettler Toledo Instrument Co., Ltd., Switzerland). ${ }^{1} \mathrm{H}$ NMR spectra were recorded in $\mathrm{D}_{2} \mathrm{O}$ as the solvent with an Avance-500 spectrometer (Bruker Company, Karlsruhe, Germany). TGA analysis was performed with a DTG$60 \mathrm{H}$ synchronous thermal analyzer (Shimadzu, Kyoto, Japan) under the conditions of a heating rate of $10{ }^{\circ} \mathrm{C} \min ^{-1}$, an $\mathrm{N}_{2}$

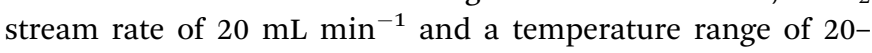
$600{ }^{\circ} \mathrm{C}$. Rheological characteristics were measured with an Anton Paar MCR302 rheometer (Anton Paar GmbH, Austria).

\subsection{Dewatering experiments}

The flocculants utilized in the dewatering experiments were synthesized by a UV-initiated template copolymerization technique. The parameters of the flocculants employed in this study are listed in Table 1 . All the flocculants were copolymerized under the optimal synthesis conditions with intrinsic viscosity as the objective for optimization. WAS samples were collected from Dadukou Drainage Co., Ltd. (Chongqing, China). The main process employed at this plant was a cyclic activated sludge system (CAST process) with a capacity of $50000 \mathrm{~m}^{3} \mathrm{~d}^{-1}$. The samples were stored under refrigeration at a temperature of $4{ }^{\circ} \mathrm{C}$ and were analyzed within $48 \mathrm{~h}$. The main characteristics of the WAS are shown in Table 2.

A programmable jar test apparatus (Wuhan Hengling Technology Ltd., China) was employed in the dewatering experiments. The efficiency of sludge dewatering was evaluated in terms of FCMC and SRF. For each type of flocculant, solutions with a concentration of $1 \mathrm{~g} \mathrm{~L}^{-1}$ were prepared with deionized water before use. A $500 \mathrm{~mL}$ sample of WAS was transferred into a $1 \mathrm{~L}$ plastic beaker, and a predetermined dosage of the flocculant was added to the system. Then, the sample was exposed to mechanical stirring conditions of $400 \mathrm{rpm}$ for $30 \mathrm{~s}$ followed by $40 \mathrm{rpm}$ for $8 \mathrm{~min}$, which were required for the complete mixing of the colloidal particles and flocculant and floc growth, respectively. ${ }^{28}$ Then, the supernatant was extracted with a syringe at a depth of $1 \mathrm{~cm}$ below the sample surface for measurements of the zeta potential ( $\zeta)$ using a Zetasizer Nano ZS90 (Malvern Instruments Ltd., Malvern, UK). The conditioned samples were filtered through a standard Buchner funnel with a $0.45 \mu \mathrm{m}$ filter paper for $3 \mathrm{~min}$ under a constant pressure of $0.6 \mathrm{MPa}$ provided by an air pump, and the volume of the filtrate was recorded every $10 \mathrm{~s}$. Then, the filter cake was placed in a DHG-9070A drying oven (Gongyi Yuhua Instrument Co., Ltd., China) and dried at a low temperature of $60{ }^{\circ} \mathrm{C}$ for $72 \mathrm{~h}$. The FCMC value was calculated from the following eqn (1):

$$
\mathrm{FCMC} \%=\frac{W_{1}-W_{2}}{W_{1}} \times 100
$$

where $W_{1}$ is the weight of the filter cake at the end of filtration and $W_{2}$ is the weight of the dried cake. The SRF value was obtained by the method described by Qi et al. and Wang et al. ${ }^{\mathbf{2 9 , 3 0}}$ using the following eqn (2):

$$
\mathrm{SRF}=\frac{2 b P A^{2}}{\mu C}
$$

where $P\left(\mathrm{~N} \mathrm{~m}^{-2}\right)$ is the filtration pressure, $A\left(\mathrm{~m}^{2}\right)$ is the filtration area, $b$ is the time-to-filtration ratio, which is the slope obtained from a plot of $t / V_{\mathrm{f}}(y)$ against $V_{\mathrm{f}}(x)$, where $V_{\mathrm{f}}$ is the volume of the filtrate $\left(\mathrm{m}^{3}\right)$ and $t$ is the filtration time (s), and $C$ is the weight of

Table 1 Flocculants utilized in the dewatering experiments

\begin{tabular}{llll}
\hline & $\begin{array}{l}\text { Intrinsic } \\
\text { viscosity } \\
\left(\mathrm{dL} \mathrm{g}^{-1}\right)\end{array}$ & $\begin{array}{l}\text { Cationic } \\
\text { degree } \\
(\%)\end{array}$ & $\begin{array}{l}\text { Content of } \\
\text { hydrophobic } \\
\text { monomers }(\%)\end{array}$ \\
\hline TPADL-7.96-25-2 & 7.96 & 25 & 2 \\
PADL-8.20-25-2 & 8.20 & 25 & 2 \\
TPAD-8.07-25-0 & 8.07 & 25 & 0 \\
PAD-8.27-25-0 & 8.17 & 25 & 0
\end{tabular}


Table 2 Main characteristics of $\mathrm{WAS}^{a}$

\begin{tabular}{|c|c|c|c|c|c|c|}
\hline Moisture content (\%) & $\mathrm{pH}$ & $\begin{array}{l}\text { Mass density } \\
\left(\mathrm{mL} \mathrm{g}^{-1}\right)\end{array}$ & $\zeta$-Potential (mV) & $\begin{array}{l}\text { Conductivity } \\
\left(\mathrm{mS} \mathrm{cm}^{-1}\right)\end{array}$ & $d_{50}(\mu \mathrm{m})$ & VSS/TSS $\left(\mathrm{mg} \mathrm{L}^{-1}\right)$ \\
\hline $98.6 \pm 0.32$ & $6.93 \pm 0.15$ & 0.936 & $-24.1 \pm 0.3$ & $1.42 \pm 0.2$ & 62.17 & $0.66 \pm 0.2$ \\
\hline
\end{tabular}

solids per unit volume of the filtrate $\left(\mathrm{kg} \mathrm{m}^{-3}\right)$, which is determined by $C=\left(1 / C_{\mathrm{i}}\right) /\left[\left(100 C_{\mathrm{i}}-\mathrm{C}_{\mathrm{f}}\right) / 100 C_{\mathrm{f}}\right]$, where $C_{\mathrm{i}}$ is the initial moisture content (\%) and $C_{\mathrm{f}}$ is the final moisture content (\%). ${ }^{31}$

\subsection{Floc properties}

The floc size distribution was measured with a BT-9300HT laser particle size analyzer (Beijing Judaohesheng Technology Co., Ltd., China). Floc breakage and recovery experiments were carried out as follows: the aforementioned flocculation process was followed by a subsequent breakage phase of $400 \mathrm{rpm}$ for $30 \mathrm{~s}$ and a recovery phase of $40 \mathrm{rpm}$ for $10 \mathrm{~min}$. The floc size after each phase was determined as mentioned above. The floc breakage and recovery factors have previously been employed to represent the relative breakage and recovery processes of flocs. ${ }^{32,33}$ The breakage factor and recovery factor were calculated using the following eqn (3) and (4), respectively:

$$
\begin{gathered}
B_{\mathrm{f}} \%=\frac{d_{2}}{d_{1}} \times 100 \\
R_{\mathrm{f}} \%=\frac{d_{3}-d_{2}}{d_{1}-d_{2}} \times 100
\end{gathered}
$$

where $d_{1}, d_{2}$ and $d_{3}$ are the floc sizes before the breakage phase, after the breakage phase and after the recovery phase, respectively. Here, the floc size is indicated by the equivalent volumetric diameter $d_{50}$, which represents the $50^{\text {th }}$ percentile floc size.

Field emission scanning electron microscopy (FESEM: Mira 3 LMU SEM system, TESCAN Company, Czech Republic) was employed to investigate the structural morphology of dry sludge cakes of conditioned samples. Image-Pro Plus software was also used to calculate the fractal dimension. ${ }^{34,35}$

\section{Results and discussion}

\subsection{Characterization of TPADL}

3.1.1 FTIR spectra of TPADL and PADL flocculants. The FTIR spectra of the synthesized TPADL and PADL are illustrated in Fig. 2. As shown in Fig. 2(a), the absorption peaks present at 3431 and $1169 \mathrm{~cm}^{-1}$ are assigned to strong stretching vibrations of the amino group and the carbonyl group of the amide moiety in AM, respectively. The peak at $1453 \mathrm{~cm}^{-1}$ is attributed to the $-\mathrm{CH}_{2}-\mathrm{N}^{+}\left(\mathrm{CH}_{3}\right)_{3}$ group of the DAC monomer. ${ }^{15}$ The absorption peak at $1667 \mathrm{~cm}^{-1}$ is due to the stretching vibrations of $-\mathrm{C}=\mathrm{O}$ groups in the AM and LA monomers, and the peak that appears at $953 \mathrm{~cm}^{-1}$ originated from the $-\mathrm{CO}-\mathrm{O}$ groups of LA monomers. $^{27}$ The spectrum of PADL is shown in Fig. 2(b). When compared with that of TPADL, it is obvious that the characteristic peaks in Fig. 2(a) are all present in Fig. 2(b) and display only a slight shift in wavenumbers. This result indicates the formation of flocculants containing the monomers AM, DAC and LA and the similarity in composition between TPADL and PADL. It is worth noting that PAAS was successfully removed and did not participate in the copolymerization.

3.1.2 ${ }^{1} \mathrm{H}$ NMR spectra of TPADL and PADL flocculants. The microstructure of the TPADL flocculant was determined by ${ }^{1} \mathrm{H}$ NMR spectroscopy, and the composition of TPADL was further defined. As seen in Fig. 3(a), the spectrum of TPADL exhibits several characteristic peaks because of the incorporation of different monomers. The sharp signal at $\delta=4.79 \mathrm{ppm}$ is attributed to the solvent $\mathrm{D}_{2} \mathrm{O}$. The signals at $\delta=1.67 \mathrm{ppm}$ (a) and $\delta=2.24 \mathrm{ppm}$ (b) prove the presence of backbone methylene groups and methine groups $\left(-\mathrm{CH}_{2}-\mathrm{CH}-\right)$, respectively. The signals at $\delta=4.58 \mathrm{ppm}(\mathrm{c})$ and $\delta=3.76 \mathrm{ppm}(\mathrm{d})$ are derived from the protons in the methylene group connected to the ammonium group in the DAC monomer. The signal at $\delta=$ $3.21 \mathrm{ppm}$ (e) corresponds to the protons in the three equivalent methyl groups of the ammonium moiety $-\mathrm{N}^{+}\left(\mathrm{CH}_{3}\right)_{3}$ in DAC. ${ }^{\mathbf{1 6 , 2 4}}$ The signal at $\delta=2.35 \mathrm{ppm}$ (f) gives evidence that the protons in the methylene group connected to the ester function in the LA monomer. The signals of the protons in the terminal methyl group in the LA monomer are present at 1.17-1.20 ppm (g). ${ }^{20,36}$ The ${ }^{1} \mathrm{H}$ NMR spectrum further confirms the successful preparation of TPADL from monomers of AM, DAC and LA.

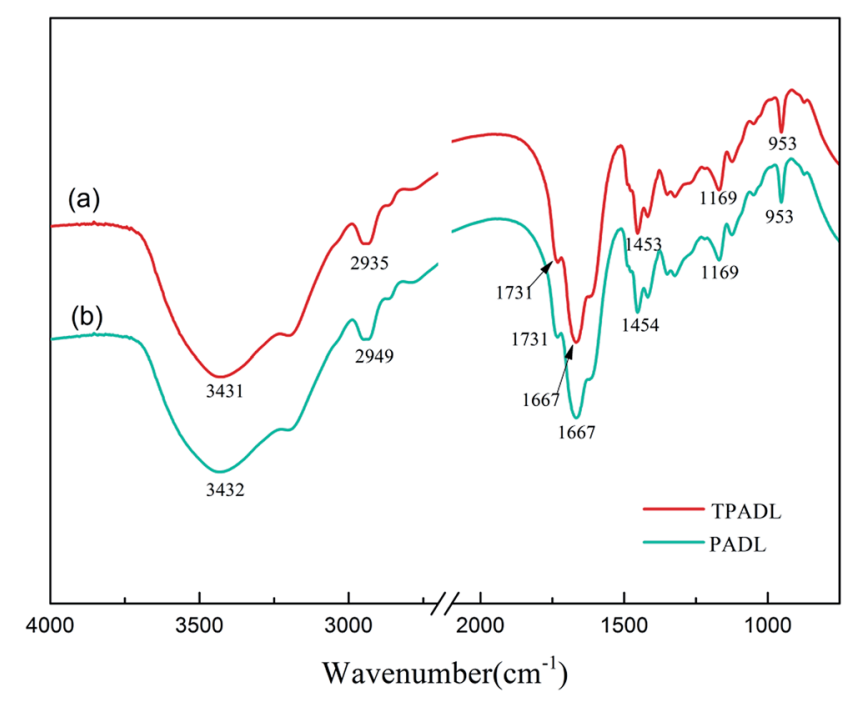

Fig. 2 FTIR spectrum of (a) TPADL and (b) PADL. 


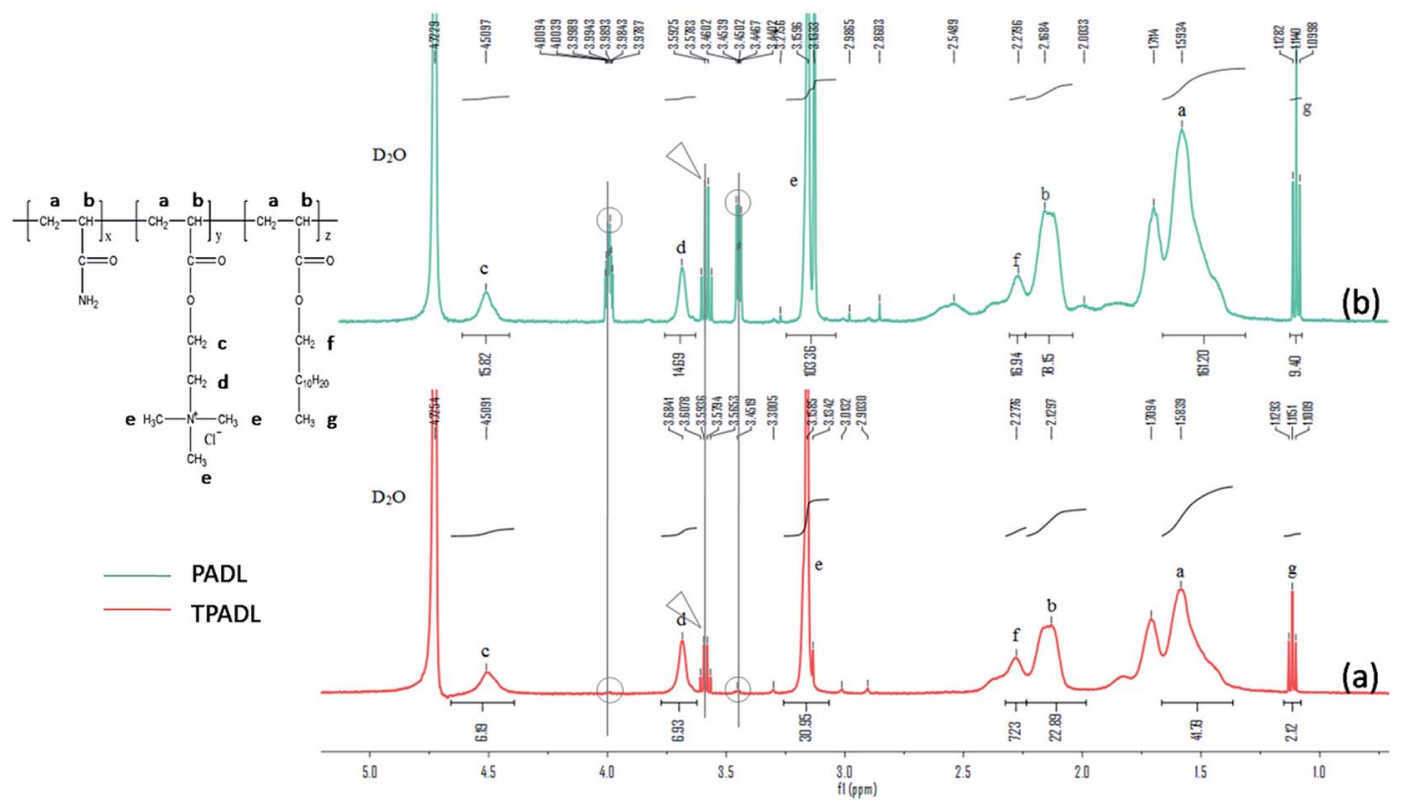

Fig. $3{ }^{1} \mathrm{H}$ NMR spectrum of (a) TPADL and (b) PADL.

The ${ }^{1} \mathrm{H}$ NMR spectrum of PADL shown in Fig. $3(\mathrm{~b})$ exhibits similar vibration signals to those of TPADL shown in Fig. 3(a), which indicates that the PADL flocculant has an identical composition to that of TPADL. However, several differences cannot be ignored, which are essential for analysis of the microblock structure. Notably, the signals detected at $3.90 \mathrm{ppm}$, $3.65 \mathrm{ppm}$ and $3.52 \mathrm{ppm}$, which are marked by circles and a triangle, are significantly weakened owing to the template copolymerization technique. According to Guan et al. and Chen et al., ${ }^{15,16}$ this phenomenon is attributed to the stereochemistry of the copolymers. The DAC monomer in the PADL polymer chain is in a disorganized sequence. This random distribution will give rise to several more resonance peaks owing to the steric interaction between different pendant groups. In contrast, the proton signals of the $-\mathrm{O}-\mathrm{CH}_{2}-(\mathrm{c}),-\mathrm{CH}_{2}-\mathrm{N}^{+}(\mathrm{d})$, and $-\mathrm{N}^{+}\left(\mathrm{CH}_{3}\right)_{3}$ (e) groups in the cationic microblock structure, which are arranged along the PAAS chain via strong intermolecular ionic bonds when synthesized, can be uniform in general owing to the steric and electrostatic repulsion of the pendant groups, and only one resonance is observed for each single group in theory. ${ }^{37,38}$ Therefore, the three marked signals are obviously weakened owing to the identical sequence of cationic units in the cationic microblock structure. Undoubtedly, this centralized distribution of cationic units would efficiently enhance the charge neutralization performance and provide a more highly stretched linear configuration of the polymer chain in solution.

3.1.3 TGA analysis. Fig. 4 shows the thermal stability of the TPADL and PADL flocculants. Three main stages of thermal decomposition are observed from the corresponding weight losses. During the initial stage, a weight loss of $7.1 \%(\mathrm{w} / \mathrm{w})$ was observed for TPADL in the temperature range of $30-200{ }^{\circ} \mathrm{C}$, whereas for PADL the values were $8.4 \%$ and $30-210{ }^{\circ} \mathrm{C}$, respectively. These losses are attributed to the evaporation of water molecules adsorded on the hydrophilic groups of the polymer chain. ${ }^{39,40}$ The second stage was observed in the ranges of $200-320^{\circ} \mathrm{C}$ and $210-340{ }^{\circ} \mathrm{C}$ with respective weight losses of $25.5 \%$ and $23.9 \%$. These losses are considered to be due to an
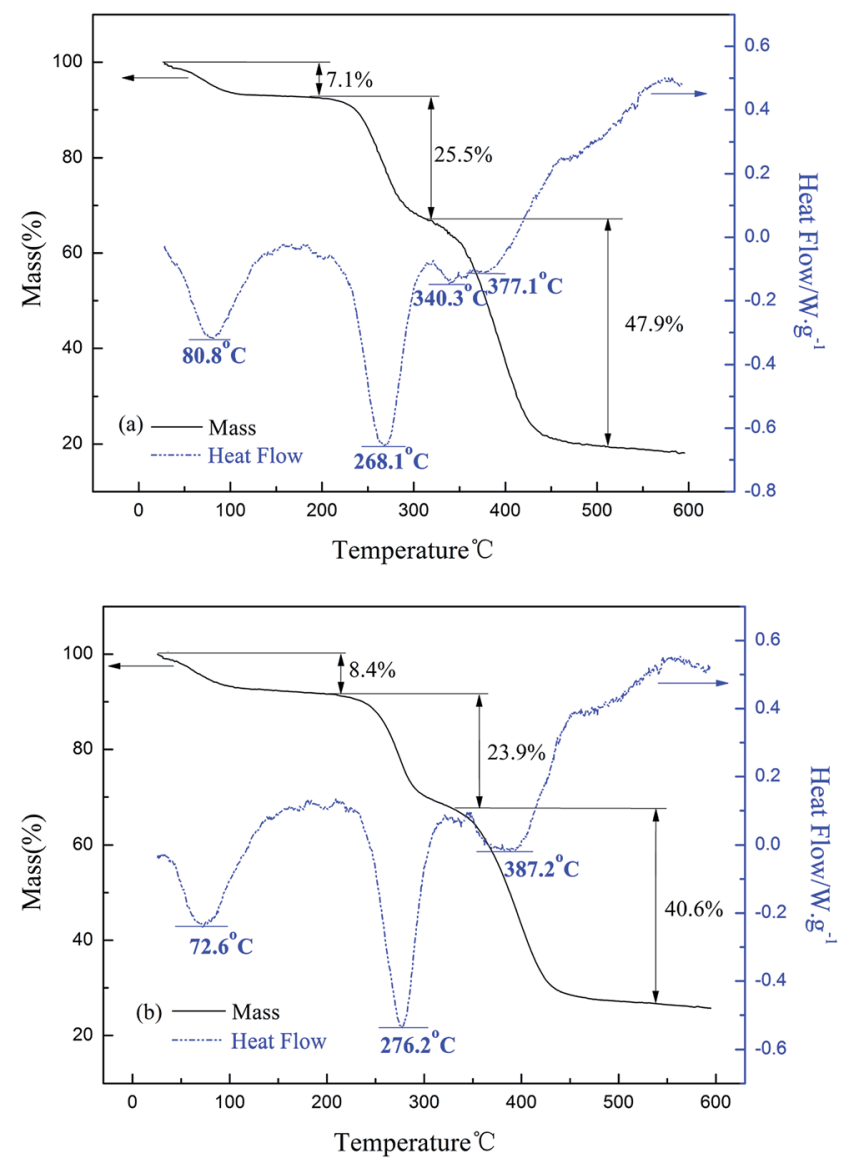

Fig. 4 TGA analysis of (a) TPADL and (b) PADL. 
imine reaction of the amide group and thermal decomposition of the hydrophobic side chain. ${ }^{41,42}$ Moreover, the similar heat absorption peaks at $268.1{ }^{\circ} \mathrm{C}$ and $276.2^{\circ} \mathrm{C}$ show the favorable thermal stability of both TPADL and PADL. ${ }^{20}$ In the final stage, the decomposition of both TPADL and PADL occurred above $320{ }^{\circ} \mathrm{C}$ with weight losses of $47.9 \%$ and $40.6 \%$, respectively. These weight losses were due to thermal decomposition of the copolymer backbone. The thermogravimetric curves reach a steady state when the temperature is above $450{ }^{\circ} \mathrm{C}$ and the final residual weight is about $20 \%$ of that of the initial sample. According to Fig. 4(a), the TPADL sample exhibits two apparent heat absorption peaks at $340.3{ }^{\circ} \mathrm{C}$ and $377.1{ }^{\circ} \mathrm{C}$, whereas only one peak at $387.2^{\circ} \mathrm{C}$ can be found for PADL in Fig. 4(b). The reason for this phenomenon can be ascribed to the unit sequence distribution of the copolymer. ${ }^{15,16}$ In this analysis, ignoring the negligible influence of the small amount of block structures of hydrophobic units, the product of template copolymerization, namely, TPADL, mainly contains cationic blocks, and the block structure of AM units displays two independent heat absorption peaks for the degradation of two kinds of backbone. However, AM and DAC units are randomly distributed in the main chain of PADL, and the decomposition of the PADL backbone is considered to be a combination of that of AM, DAC and LA units. Thus, only one heat absorption peak can be observed. Li et al. and Zhang et al. also reported similar findings about the independent heat absorption peaks of copolymers with microblock structures. ${ }^{25,26}$ The findings of thermal analysis further confirm the presence of cationic microblock structures in TPADL.

3.1.4 Apparent viscosity of TPADL, PADL and TPAD. In general, measurements of apparent viscosity are employed as a convenient and reliable method for investigating the associative performance of amphiphilic copolymers in aqueous solution. ${ }^{19}$ In this study, the apparent viscosity was determined as a function of the polymer concentration of TPADL, PADL and TPAD. As shown in Fig. 5, the apparent viscosity of all three copolymers increased linearly with an increase in concentration. The apparent viscosities of TPADL and PADL were almost

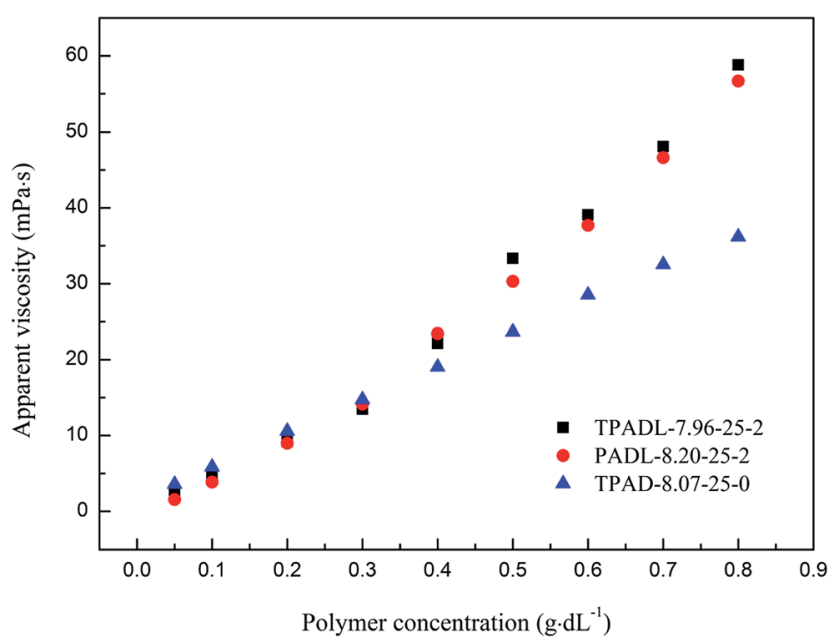

Fig. 5 Apparent viscosity as a function of the polymer concentration for TPADL, PADL and TPAD. the same but were slightly lower than that of TPAD in the beginning. However, with a continuous increase in concentration to above about $0.3 \mathrm{~g} \mathrm{dL}^{-1}$ the apparent viscosity of TPADL and PADL increased markedly, whereas that of TPAD maintained a linear increase, as before. This phenomenon is attributed to a transition between intra- and inter-molecular association of hydrophobic units..$^{19}$ Intramolecular association is dominant in solvents when the polymer concentration is below the CAC (critical association concentration). Intramolecular association will lead to the polymer chain adopting a curled configuration to reduce its hydrodynamic volume. Thus, TPADL and PADL were observed to have comparatively low apparent viscosity. On the contrary, when the polymer concentration is above the CAC intermolecular association is dominant in solvents and forms a physical network structure of polymer chains with a large hydrodynamic volume. Thus, very high apparent viscosity is observed. According to the changes in apparent viscosity, the CAC of TPADL and PADL is about $0.3 \mathrm{~g}$ $\mathrm{dL}^{-1}$. The apparent viscosities of TPADL and PADL finally reached $58.795 \mathrm{mPa} \mathrm{s}^{-1}$ and $56.674 \mathrm{mPa} \mathrm{s}^{-1}$, respectively, whereas that of TPAD was only $36.189 \mathrm{mPa} \mathrm{s}^{-1}$. All these results are attributed to the introduction of the hydrophobic group on the polymer chain, and this reversible physical cross-linking of the network can facilitate the floc recovery process. It is worth noting that the apparent viscosity of TPADL was slightly higher than that of PADL. This phenomenon occurred because of the utilization of a template copolymerization technique. The concentrated distribution of charge provided a more highly stretched linear configuration of the polymer chain in solution, which was beneficial for the occurrence of intermolecular association. This result provides further indirect evidence of the formation of a cationic microblock structure.

\subsection{Dewatering performance}

As illustrated in Fig. 6, the FCMC and SRF values of samples conditioned by a series of flocculants were determined to evaluate the dewaterability, and the zeta-potential was also determined to further investigate the flocculation mechanism. The purpose of this part of the study is to accurately evaluate the effect of the synergistic function of the cationic microblock structure and hydrophobic association on the dewaterability of sludge.

Fig. 6(a) shows the respective results for the FCMC values of samples conditioned by four types of flocculant. The FCMC value was initially reduced by the addition of each type of flocculant. The lowest FCMC (about 65\%), which was achieved with TPADL at a dosage of $1 \mathrm{mg} \mathrm{g}^{-1}$, was lower than the values of $73.1 \%$ and $67.8 \%$ reported by Liao et al. ${ }^{19}$ and Zheng et al. ${ }^{27}$ respectively. However, the FCMC gradually increased again with further dosages in excess of the optimum value $\left(1 \mathrm{mg} \mathrm{g}^{-1}\right)$. The FCMC values of the conditioned samples were in the following order: TPADL $<$ PADL $<$ TPAD $<$ PAD. The FCMC values of both PADL and TPAD were below 70\%. According to Feng et al. and Zhang et al., the SRF value is accepted as an indicator for the evaluation of sludge filtration performance. ${ }^{43,44}$ In general, a lower SRF value indicates superior dewatering performance in 

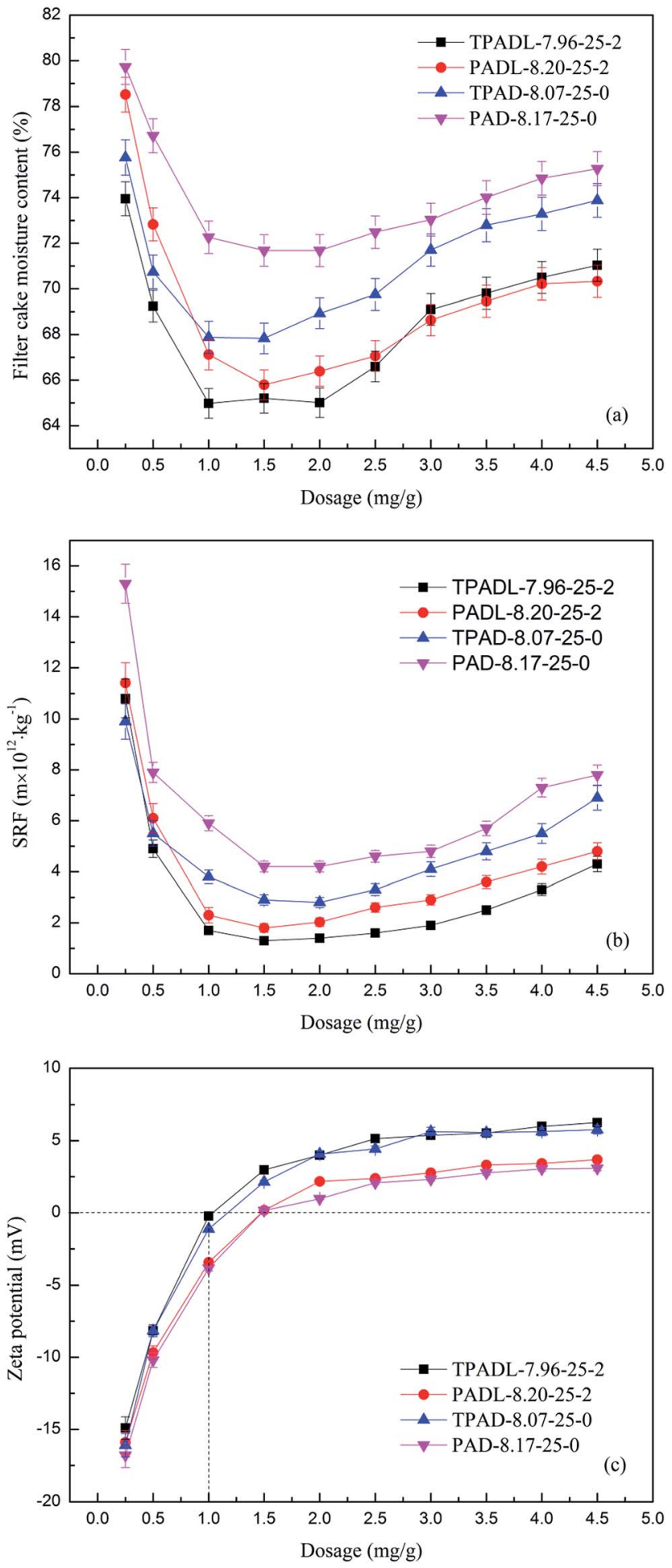

Fig. 6 Effect of flocculant dosage on (a) FCMC, (b) SRF and (c) $\zeta-$ potential of conditioned sludge.

mechanical dehydration. ${ }^{43}$ The SRF values of the conditioned samples are shown in Fig. 6(b). The results for the SRF values show a similar trend to those of the FCMC values. The SRF value first decreased in the dosage range of $0.25 \mathrm{mg} \mathrm{g}^{-1}$ to $1.5 \mathrm{mg} \mathrm{g}^{-1}$, and when the amount of flocculant added exceeded the optimal dosage (about $1.5 \mathrm{mg} \mathrm{g}^{-1}$ ) the SRF value began to rise. On the basis of the SRF results, TPADL exhibited better performance than the flocculants used for the other three samples over most of the dosage range, and the optimal SRF value of approximately $1.4 \times 10^{13} \mathrm{~m} \mathrm{~kg}^{-1}$ was achieved with TPADL at a dosage of $1.5 \mathrm{mg} \mathrm{g}^{-1}$, which was lower than that reported by Wang et al. ${ }^{7}$

The superiority of the dewatering performance of TPADL revealed that the synergistic effect of hydrophobic association and the cationic microblock structure can facilitate the dewatering of sludge. The zeta potentials of the conditioned samples are shown in Fig. 6(c). From these curves, with an increase in the flocculant dosage the zeta potential rapidly increased from negative values $(-24.1 \mathrm{mV})$ to the isoelectric point. The isoelectric point was reached at a dosage of about $1 \mathrm{mg} \mathrm{g}^{-1}$ for the cationic microblock copolymers and $1.5 \mathrm{mg} \mathrm{g}^{-1}$ for the ordinary cationic copolymers. This phenomenon reveals that the flocculants with a cationic microblock structure exhibited higher charge neutralization ability than that of flocculants with a random distribution of cations. The charge neutralization function could effectively reduce the repulsive effect of negative charge and compress the electrical double layer of colloidal sludge particles. The cationic microblock segment can be tightly adsorbed on the negatively charged surface of colloidal particles to reduce the hydrated shell by lowering the surface tension of water. ${ }^{16}$ As a result, a lower moisture content was achieved. Furthermore, this kind of concentrated distribution of cations can provide an expanded linear configuration of the polymer chain in the sludge solution. This elongated stretched shape and the physical network structure formed by intermolecular hydrophobic association can significantly increase the probability of collisions between the polymer chain and colloidal particles, so that the bridging effect is strengthened to help the aggregation of the particles to form giant and compact flocs. ${ }^{31}$ More details of the floc properties will be discussed in Section 3.3. Because the optimal dosage did not coincide with the exact isoelectric point, charge neutralization was not the only dominant mechanism but the bridging absorption effect also played an important role during the flocculation process. ${ }^{45}$

With further increases in dosage beyond the isoelectric point, the zeta potential continued to increase to positive values of $5 \mathrm{mV}$ for the templated copolymers and approximately $2.5 \mathrm{mV}$ for the normal cationic copolymers. In comparison with the phase before the isoelectric point, the rate of increase in the zeta potential significantly slowed down. This phenomenon can be explained by the electrostatic patching effect. ${ }^{46}$ In a colloidal system, when, or even after, the isoelectric point is reached, the floc surface may absorb several cationic microblock segments to form positively charged patches. Nevertheless, negatively charged surfaces are still present and exposed to the colloidal system. This kind of cationic patch can further absorb other negatively charged surfaces of flocs to aggregate into larger flocs.

During the entire dewatering test, TPADL displayed superior dewatering performance to those of the PADL and TPAD flocculants and significantly better performance than that of PAD. It can be confirmed that both hydrophobic association and a cationic microblock structure are suitable for the dewatering 
of sludge, and the reason for the superiority of TPADL is because of the synergistic effect of these factors.

\subsection{Floc properties}

The floc properties are closely related to the flocculation behavior in a sludge system. In order to obtain an in-depth understanding of the dewatering process caused by the synergistic effect of the cationic microblock structure and hydrophobic association, we further analyzed the floc properties of sludge in terms of the floc size distribution and breakage and recovery factors, as well as the surface morphology of the dried sludge.

3.3.1 Floc size distribution. Fig. 7 shows the floc size distribution in the stable state before breakage. The floc sizes $\left(d_{50}\right)$ were $360.382 \mu \mathrm{m}, 403.191 \mu \mathrm{m}, 289.352 \mu \mathrm{m}$ and $314.514 \mu \mathrm{m}$ for TPADL, PADL, TPAD and PAD, respectively. From the measurements of floc sizes, the introduction of hydrophobic association significantly increased the floc diameter of conditioned colloidal particles of WAS. This was because of an enhancement of the bridging absorption effect. The intermolecular hydrophobic association successfully formed a physical network structure that significantly increased the likelihood of collisions between polymer chains and colloidal particles. A number of previous studies have proposed that an enhancement in bridging absorption capability could result in a more substantial increase in floc size and thus a larger floc diameter. ${ }^{32,47}$ It is worth noting that the cationic microblock structure formed by the template copolymerization technique exerted a negative effect on floc development. This could be attributed to the decrease in the repulsive force between colloidal particles. The concentration of cationic units can considerably enhance the charge neutralization ability of the flocculant to effectively reduce charge repulsion and compress the electrical double layer of colloidal sludge particles. Hence, the higher charge neutralization ability makes flocs have a smaller but more compact structure. For the samples conditioned by TPADL, both hydrophobic association and stronger charge neutralization took effect together to form giant and compact flocs.
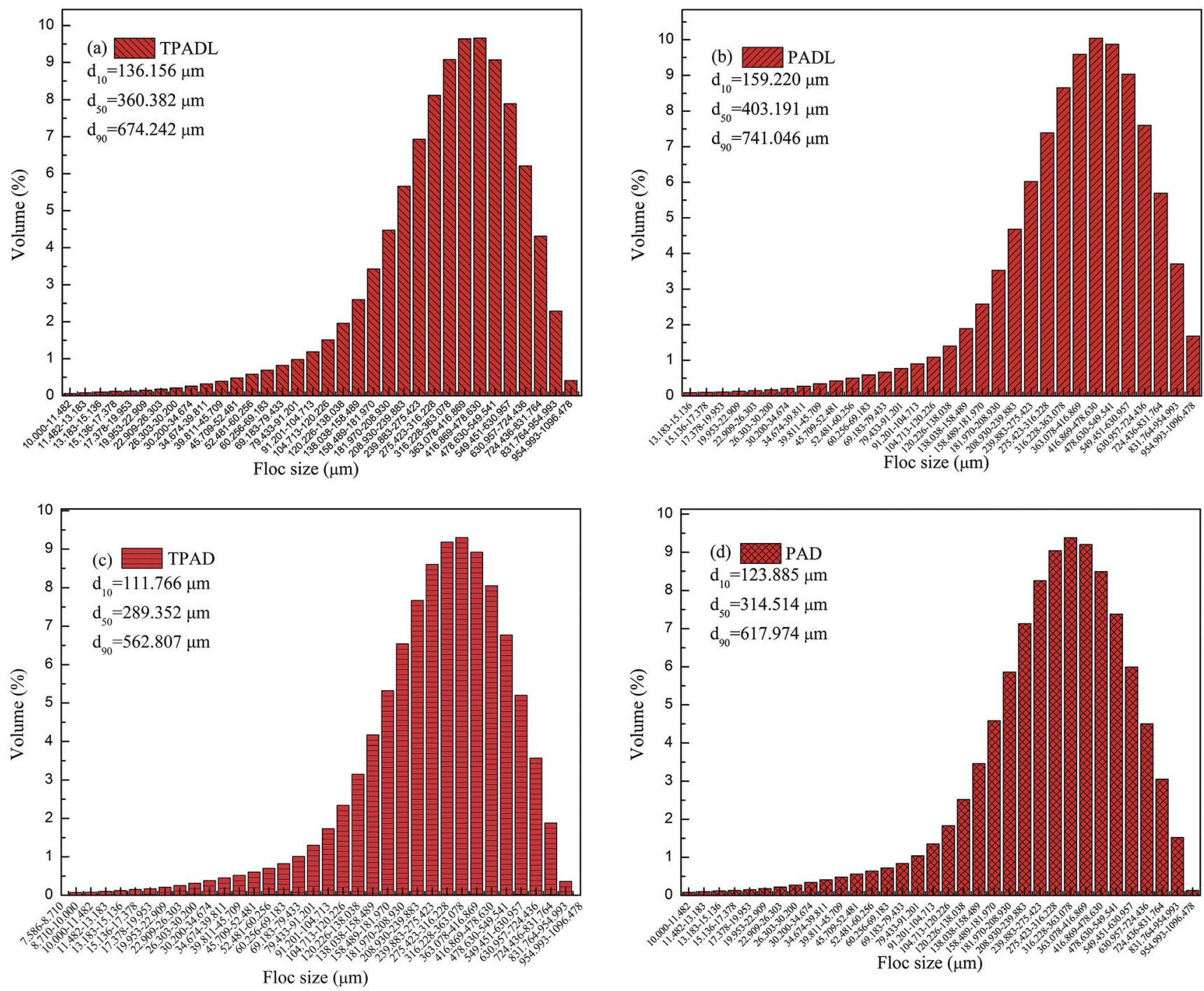

Fig. 7 Floc size $\left(d_{50}\right)$ distribution of samples conditioned by (a) TPADL, (b) PADL, (c) TPAD and (d) PAD. 
Table 3 Floc sizes $\left(d_{50}\right)$ of conditioned samples before and after the recovery phase

\begin{tabular}{lll}
\hline Coagulant & $\begin{array}{l}\text { After breakage } \\
(\mu \mathrm{m})\end{array}$ & $\begin{array}{l}\text { After recovery } \\
(\mu \mathrm{m})\end{array}$ \\
\hline TPADL-7.96-25-2 & 200.276 & 234.633 \\
PADL-8.20-25-2 & 193.661 & 236.889 \\
TPAD-8.07-25-0 & 173.113 & 185.225 \\
PAD-8.17-25-0 & 138.272 & 151.615 \\
\hline
\end{tabular}

Table 4 Breakage factor and recovery factor of conditioned samples (\%)

\begin{tabular}{lll}
\hline Coagulant & $\begin{array}{l}\text { Breakage factor } \\
(\%)\end{array}$ & $\begin{array}{l}\text { Recovery factor } \\
(\%)\end{array}$ \\
\hline TPADL-7.96-25-2 & 55.57 & 21.46 \\
PADL-8.20-25-2 & 48.03 & 20.63 \\
TPAD-8.07-25-0 & 59.83 & 10.42 \\
PAD-8.17-25-0 & 43.96 & 7.57
\end{tabular}

3.3.2 Floc breakage and recovery factors. Table 3 shows the floc sizes $\left(d_{50}\right)$ of the conditioned samples before and after the recovery phase, respectively. Fig. S2 and S3 in the $\mathrm{ESI}_{\dagger}^{\dagger}$ present the detailed floc size distributions in the two phases.
Table 4 shows the breakage and recovery factors for four flocculants at the optimal dosage. The breakage factor and recovery factor were calculated by eqn (3) and (4), respectively, which were mentioned before in Section 2.3. It can be seen that the introduction of the cationic microblock structure effectively increased both the breakage factor and the recovery factor. The reason for the increase in the breakage factor could be attributed to the enhancement of the bridging absorption effect. It is widely accepted that the polymer bridging effect can increase the strength of flocs. ${ }^{32}$ In our specific study, the concentrated distribution of cationic units can give rise to an expanded linear configuration of the polymer chain, which is beneficial for the occurrence of the bridging absorption effect in a flocculation system. Furthermore, this kind of cationic microblock segment could be tightly adsorbed on the negatively charged surface of sludge particles with fewer wasted cationic units, and the stronger electrostatic attraction force enables higher shearing resistance. This can explain how the breakage factor was increased by the cationic microblock structure. However, hydrophobic association exerted a comparatively negative effect on the breakage factor. Hydrophobic association is a kind of weak but reversible physical combination of hydrophobic groups on the polymer chain. When intermolecular hydrophobic association occurs, the physical network structure of the
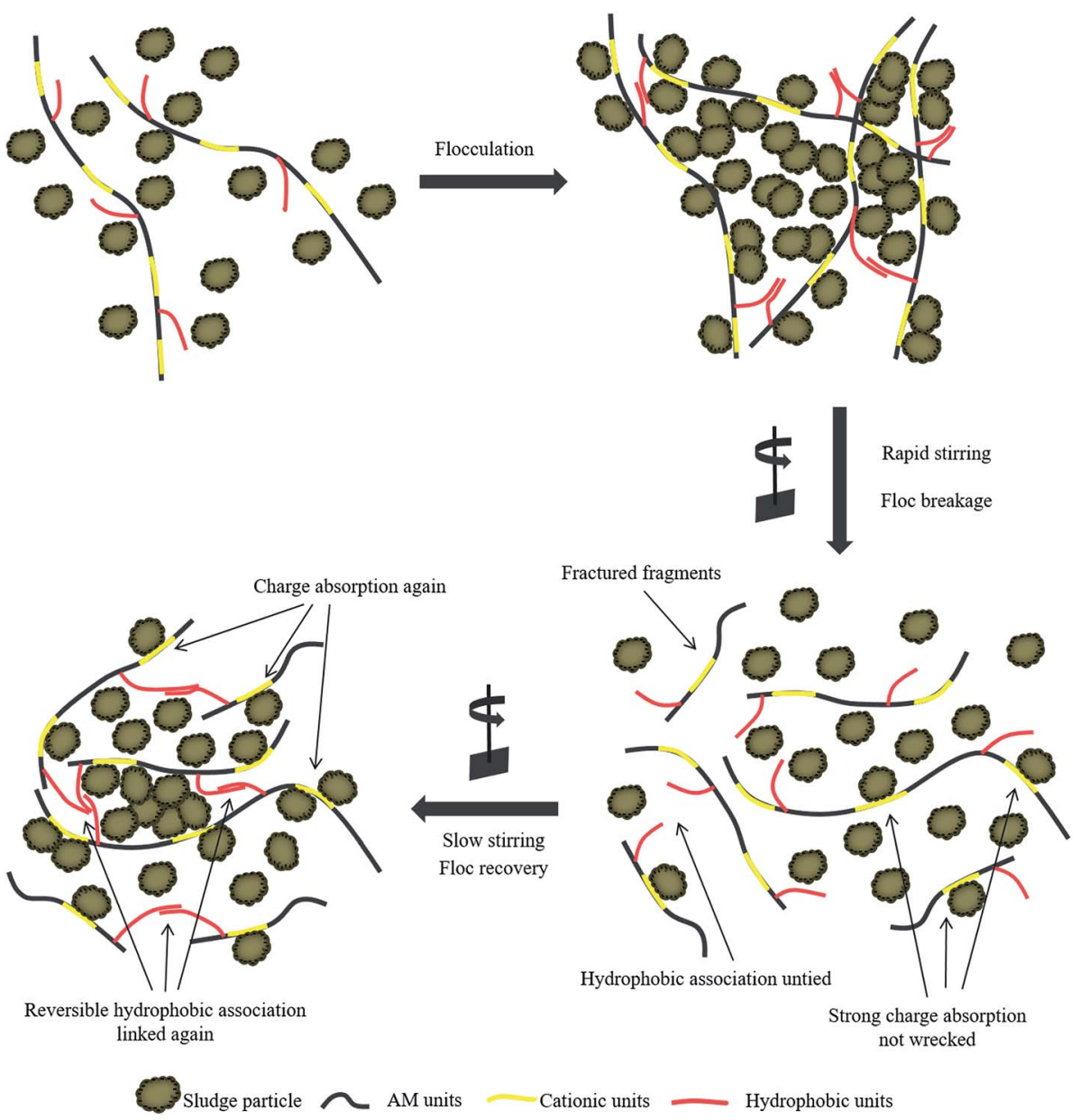

Fig. 8 Possible regeneration mechanism of TPADL. 
polymer chain can significantly reinforce the bridging absorption effect to form larger flocs. However, this kind of physical bond between hydrophobic groups is too weak to resist high shearing forces. Once a high shearing rate was applied to the flocculation system, the effect of hydrophobic association was easily overcome and giant flocs broke up into normal-sized flocs. Thus, hydrophobic association exerted a negative effect on the breakage factor. With the reintroduction of slow stirring after breakage, an apparent phenomenon of floc regrowth could be observed for all the conditioned samples except for that conditioned by PAD. When compared with the polymers with a random distribution of cations, the polymers with a cationic microblock structure displayed a comparatively high recovery ability. The recovery factors increased from $20.63 \%$ and $7.57 \%$
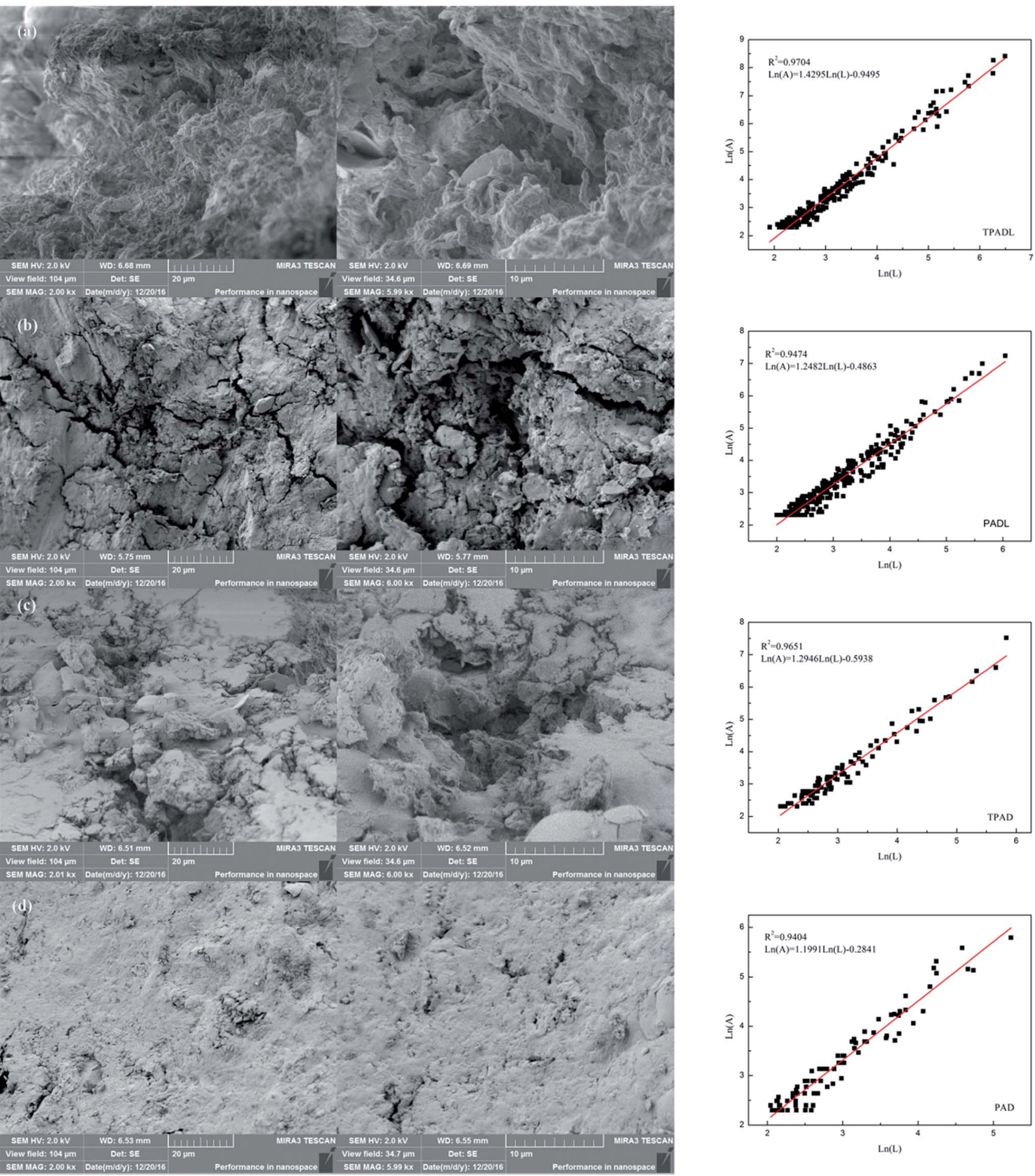

Fig. 9 SEM photos of structural morphology of samples conditioned by (a) TPADL, (b) PADL, (c) TPAD and (d) PAD. 
for PADL and PAD to $21.46 \%$ and $10.42 \%$ for TPADL and TPAD, respectively. This phenomenon is also proof that polymers with a concentrated distribution of cations exhibited stronger charge neutralization than those with a random distribution. Aguilar et al. and Jarvis et al. also mentioned the superior recovery capability of flocs that were formed mainly by the charge neutralization effect. ${ }^{\mathbf{4 8 , 4 9}}$

According to the results for the recovery factor in Table 4, the introduction of hydrophobic association led to extraordinary improvements in the recovery ability of flocs. The recovery factors of TPAD and PAD were only $10.42 \%$ and $7.57 \%$, but for TPADL and PADL the recovery factor significantly increased to $21.46 \%$ and $20.63 \%$, respectively. The increase in the recovery factor was because of intermolecular hydrophobic association, which is a reversible association linked by physical bonding rather than chemical bonding. Although this weak physical bond will be easily destroyed under conditions of a high shearing force, once a slow stirring rate is applied to the flocculation system again after the breakage phase, this kind of physical link can rapidly reform to reaggregate the shattered flocs into large flocs. A possible mechanism of the regeneration of flocs by TPADL is displayed in Fig. 8.

In summary, the cationic microblock structure is beneficial for floc strength, and the synergistic effect of the cationic microblock structure and hydrophobic association can significantly improve the regeneration capability of flocs; hydrophobic association plays the crucial role in the regeneration process of flocs.

3.3.3 Structural morphology of sludge cake. In order to further analyze the floc structure, the structural morphology and fractal dimension of dry conditioned samples were also measured. Filter medium blinding and cake blinding are accepted to be the two main difficulties in the dewatering of sludge, i.e., the filter cake or filter medium is blocked by smallscale solids. ${ }^{6}$ Qi et al. also indicate that clogging of the pores of the filter cake is predominantly responsible for the impairment of filtration instead of blinding of the filter medium, which implies that filtration is mostly governed by the cake layer once it is formed and the structure of the sludge cake plays a vital role in the dewaterability of sludge. ${ }^{30}$ From the results of SEM analysis shown in Fig. 9, the sample conditioned by PAD had a smoother surface than the samples conditioned by PADL and TPAD. In particular for the sample conditioned by TPADL, there were even slit-like structures that formed on the cake surface after filtration. The linear correlation coefficient between the logarithm of the projected area $(A)$ and the characteristic length $(L)$ for all samples was greater than 0.94 , and the average fractal dimension was 1.4295, 1.2482, 1.2946 and 1.1991 for samples conditioned by TPADL, PADL, TPAD and PAD, respectively. The results for the fractal dimension also demonstrate the morphological characteristics. The introduction of the cationic microblock structure and hydrophobic association promoted the formation of pores and a channel-like structure. After evaporation, the pores and channel structure preserved on the sludge surface led to a higher value of the fractal dimension. The formation of this kind of channel-like structure is attributed to the increase in floc strength. During the flocculation process, colloidal particles of WAS were tightly linked and overlapped as a result of the enhancements in bridging absorption and charge neutralization ability. The compact and high-strength network structure performed a function like that of a framework during pressure dewatering. This framework structure effectively helped the cake to form the channel-like structure and maintain the smooth outflow of water under pressure, so that the porous filter cake became a favorable draining medium for the release of water and contributed to improvements in dewaterability.

\section{Conclusions}

A novel hydrophobically modified polyacrylamide containing a cationic microblock structure, which was denoted as TPADL, was utilized as a flocculant in the conditioning of WAS to improve the dewaterability. The chemical structures were characterized using advanced instrumentation, and the dewatering performance, as well as the flocculation mechanism, was subjected to comparative study. The major conclusions of the present study were as follows:

(a) The results of FTIR and ${ }^{1} \mathrm{H}$ NMR spectroscopy indicate the successful preparation of TPADL from monomers of AM, DAC and LA. Moreover, the obvious weakening of the interference signal peak in the ${ }^{1} \mathrm{H}$ NMR spectrum confirmed the presence of the specific identical cationic sequence in the cationic microblock structure, which is favorable for higher charge neutralization ability. TGA analysis revealed acceptable thermal stability, and the two apparent stages of heat absorption peaks further demonstrated the existence of the cationic microblock structure. Measurements of the apparent viscosity indicated that the introduction of hydrophobic groups can help to form a reversible physically cross-linked network structure, which is beneficial for bridging absorption ability and the recovery ability of flocs.

(b) Dewatering experiments confirmed the superiority of TPADL. The FCMC and SRF values were $64.98 \%$ and $1.3 \times 10^{12}$ $\mathrm{m} \mathrm{kg}^{-1}$, respectively, for TPADL at the optimal dosage of $1.5 \mathrm{mg}$ $\mathrm{g}^{-1}$ DS, which were lower than the values for the samples conditioned by other flocculants employed for comparison. The synergistic function of the cationic microblock structure and hydrophobic association effectively improved the dewaterability of WAS. The results for the zeta potential also revealed that the concentrated distribution of cations enabled stronger charge neutralization than the random distribution of cations in the dewatering test.

(c) According to the results for the floc size distribution, the hydrophobic association effect can markedly increase the floc size, and the cationic microblock structure can help to form a more compact floc structure. Moreover, the breakage and recovery factors significantly increased because of this synergistic function. The stronger floc structure can function as a skeleton structure to form pores and a channel-like structure in the filter cake, which make it a favorable draining medium for the release of water.

These results may be useful in the development of new highperformance flocculants and the in-depth study of the dewatering behavior of WAS. 


\section{Conflict of interest}

The authors declare no competing financial interest.

\section{Abbreviations}

\begin{tabular}{|c|c|}
\hline WAS & Waste activated sludge \\
\hline WWTP & Wastewater treatment plant \\
\hline UV & Ultraviolet \\
\hline CPAM & Cationic polyacrylamide \\
\hline DAC & $\begin{array}{l}2 \text {-(Acryloyloxy)- } N \text {-benzyl- } N, N \text {-dimethylethanaminium } \\
\text { chloride }\end{array}$ \\
\hline LA & Lauryl acrylate \\
\hline $\mathrm{AM}$ & Acrylamide \\
\hline PAAS & Sodium polyacrylate \\
\hline V-044 & $\begin{array}{l}\left.2,2^{\prime} \text {-Azobis[2-(2-imidazolin-2-yl)propane }\right] \\
\text { dihydrochloride }\end{array}$ \\
\hline${ }^{1} \mathrm{H}$ & ${ }^{1} \mathrm{H}$ nuclear magnetic resonance \\
\hline \multicolumn{2}{|l|}{ NMR } \\
\hline FTIR & Fourier transform infrared spectroscopy \\
\hline TGA & Thermogravimetric analysis \\
\hline SEM & Scanning electron microscopy \\
\hline FCMC & Filter cake moisture content \\
\hline SRF & Specific resistance to filtration \\
\hline VSS/ & Volatile suspended solids/total suspended solids \\
\hline \multicolumn{2}{|l|}{ TSS } \\
\hline TPADL & Templated poly(AM-DAC-LA) \\
\hline PADL & Poly(AM-DAC-LA) \\
\hline TPAD & Templated poly(AM-DAC) \\
\hline PAD & Poly(AM-DAC) \\
\hline$d_{50}$ & Median particle size \\
\hline CAC & Critical association concentration \\
\hline
\end{tabular}

\section{Acknowledgements}

This research was supported by the National Natural Science Foundation of China (Project No. 21677020 and 21477010).

\section{References}

1 D. Panepinto, S. Fiore, G. Genon and M. Acri, J. Cleaner Prod., 2016, 137, 1323-1329.

2 E. Farno, J. C. Baudez, R. Parthasarathy and N. Eshtiaghi, Chem. Eng. J., 2016, 304, 362-368.

3 P. Li, H. Li, J. Li, X. Guo, J. Liu and B. Xiao, Bioresour. Technol., 2016, 221, 31-36.

4 Q. H. Zhang, W. N. Yang, H. H. Ngo, W. S. Guo, P. K. Jin, M. Dzakpasu, S. J. Yang, Q. Wang, X. C. Wang and D. Ao, Environ. Int., 2016, 92-93, 11-22.

5 X. Guo, J. Yang, Y. Liang, J. Liu and B. Xiao, Bioprocess Biosyst. Eng., 2014, 37, 553-560.

6 B. Cao, W. Zhang, Q. Wang, Y. Huang, C. Meng and D. Wang, Water Res., 2016, 105, 615-624.

7 L. Wang, L. Wang, W. Li, D. He, H. Jiang, X. Ye, H. Yuan, N. Zhu and H. Yu, Chem. Eng. Sci., 2014, 116, 228-234.
8 W. Zhang, P. Yang, X. Yang, Z. Chen and D. Wang, Bioresour. Technol., 2015, 181, 247-253.

9 W. Zhang, B. Cao, D. Wang, T. Ma, H. Xia and D. Yu, Water Res., 2016, 88, 728-739.

10 Y. Shi, J. Yang, W. Yu, S. Zhang, S. Liang, J. Song, Q. Xu, N. Ye, S. He, C. Yang and J. Hu, Chem. Eng. J., 2015, 270, 572-581.

11 M. Garrido-Baserba, M. Molinos-Senante, J. M. AbelleiraPereira, L. A. Fdez-Güelfo, M. Poch and F. HernándezSancho, J. Cleaner Prod., 2015, 107, 410-419.

12 Y. Li, X. Yuan, Z. Wu, H. Wang, Z. Xiao, Y. Wu, X. Chen and G. Zeng, Chem. Eng. J., 2016, 303, 636-645.

13 H. Luo, X. Ning, X. Liang, Y. Feng and J. Liu, Bioresour. Technol., 2013, 139, 330-336.

14 S. Agarwal, M. Abu-Orf and J. T. Novak, Water Res., 2005, 39, 1301-1310.

15 Q. Guan, H. Zheng, J. Zhai, C. Zhao, X. Zheng, X. Tang, W. Chen and Y. Sun, Ind. Eng. Chem. Res., 2014, 53, 56245635.

16 W. Chen, H. Zheng, Q. Guan, H. Teng, C. Zhao and C. Zhao, Ind. Eng. Chem. Res., 2016, 55, 2892-2902.

17 B. Bolto and J. Gregory, Water Res., 2007, 41, 2301-2324.

18 K. E. Lee, N. Morad, B. T. Poh and T. T. Teng, Desalination, 2011, 270, 206-213.

19 Y. Liao, H. Zheng, L. Qian, Y. Sun, L. Dai and W. Xue, Ind. Eng. Chem. Res., 2014, 53, 11193-11203.

20 Z. L. Yang, B. Y. Gao, C. X. Li, Q. Y. Yue and B. Liu, Chem. Eng. J., 2010, 161, 27-33.

21 D. A. Z. Wever, F. Picchioni and A. A. Broekhuis, Prog. Polym. Sci., 2011, 36, 1558-1628.

22 K. C. Taylor and H. A. Nasr-El-Din, J. Pet. Sci. Eng., 1998, 19, 265-280.

23 Y. J. Feng, L. Billon, B. Grassl, G. Bastiat, O. Borisov and J. Francois, Polymer, 2005, 46, 9283-9295.

24 H. Zheng, Y. Sun, C. Zhu, J. Guo, C. Zhao, Y. Liao and Q. Guan, Chem. Eng. J., 2013, 234, 318-326.

25 X. Li, H. Zheng, B. Gao, Y. Sun, B. Liu and C. Zhao, Chemosphere, 2017, 167, 71-81.

26 Z. Zhang, H. Zheng, F. Huang, X. Li, S. He and C. Zhao, Ind. Eng. Chem. Res., 2016, 55, 9819-9828.

27 H. Zheng, Y. Sun, J. Guo, F. Li, W. Fan, Y. Liao and Q. Guan, Ind. Eng. Chem. Res., 2014, 53, 2572-2582.

28 K. V. Gorin, Y. E. Sergeeva, V. V. Butylin, A. V. Komova, V. M. Pojidaev, G. U. Badranova, A. A. Shapovalova, I. A. Konova and P. M. Gotovtsev, Bioresour. Technol., 2015, 193, 178-184.

29 J. Wang, S. Yuan, Y. Wang and H. Yu, Water Res., 2013, 47, 2643-2648.

30 Y. Qi, K. B. Thapa and A. F. A. Hoadley, Chem. Eng. J., 2011, 171, 373-384.

31 M. Niu, W. Zhang, D. Wang, Y. Chen and R. Chen, Bioresour. Technol., 2013, 144, 337-343.

32 P. Jarvis, B. Jefferson, J. Gregory and S. A. Parsons, Water Res., 2005, 39, 3121-3137.

33 P. Jarvis, B. Jefferson and S. A. Parsons, Environ. Sci. Technol., 2005, 39, 2307-2314. 
34 G. Zhu, H. Zheng, Z. Zhang, T. Tshukudu, P. Zhang and X. Xiang, Chem. Eng. J., 2011, 178, 50-59.

35 H. Zheng, G. Zhu, S. Jiang, T. Tshukudu, X. Xiang, P. Zhang and Q. He, Desalination, 2011, 269, 148-156.

36 G. O. Yahaya, A. A. Ahdab, S. A. Ali, B. F. Abu-Sharkh and E. Z. Hamad, Polymer, 2001, 42, 3363-3372.

37 H. S. Samanta and S. K. Ray, Carbohydr. Polym., 2014, 99, 666-678.

38 Z. Abdollahi, M. Frounchi and S. Dadbin, J. Ind. Eng. Chem., 2011, 17, 580-586.

39 Y. Liu, C. Lin and Y. Wu, J. Hazard. Mater., 2007, 146, 255261.

40 A. Gök, M. Omastová and J. Prokeš, Eur. Polym. J., 2007, 43, 2471-2480.

41 J. Ma, P. Cui, L. Zhao and R. Huang, Eur. Polym. J., 2002, 38, 1627-1633.

42 X. Wan, Y. Li, X. Wang, S. Chen and X. Gu, Eur. Polym. J., 2007, 43, 3655-3661.
43 X. Feng, J. Deng, H. Lei, T. Bai, Q. Fan and Z. Li, Bioresour. Technol., 2009, 100, 1074-1081.

44 Z. Zhang, S. Xia and J. Zhang, Water Res., 2010, 44, 30873092.

45 K. A. S. Meraz, S. M. P. Vargas, J. T. L. Maldonado, J. M. C. Bravo, M. T. O. Guzman and E. A. L. Maldonado, Chem. Eng. J., 2016, 284, 536-542.

46 W. Chen, H. Zheng, H. Teng, Y. Wang, Y. Zhang, C. Zhao and Y. Liao, PLoS One, 2015, 10, e137116.

47 Y. X. Zhao, B. Y. Gao, Q. B. Qi, Y. Wang, S. Phuntsho, J. H. Kim, Q. Y. Yue, Q. Li and H. K. Shon, J. Hazard. Mater., 2013, 258-259, 84-92.

48 M. I. Aguilar, J. Sáez, M. Lloréns, A. Soler and J. F. Ortuño, Water Res., 2003, 37, 2233-2241.

49 P. Jarvis, B. Jefferson and S. A. Parsons, Water Res., 2006, 40, 2727-2737. 